Please do not remove this page

RMIT

UNIVERSITY

\title{
Photonic instantaneous frequency measurement using non-linear optical mixing
}

Sarkhosh, Niusha; Emami, Hossein; Bui, Lam; Mitchell, Arnan

https://researchrepository.rmit.edu.au/esploro/outputs/9921863715401341/filesAndLinks?institution=61RMIT_INST\&index=null

Sarkhosh, N., Emami, H., Bui, L., \& Mitchell, A. (2008). Photonic instantaneous frequency measurement using non-linear optical mixing. Microwave Symposium Digest, 2008, IEEE MTT-S International, 599-601.

https://doi.org/10.1109/MWSYM.2008.4633237

Published Version: https://doi.org/10.1109/MWSYM.2008.4633237

Repository homepage: https://researchrepository.rmit.edu.au

(C) 2008 IEEE

Downloaded On 2023/04/26 23:06:55 +1000

Please do not remove this page 


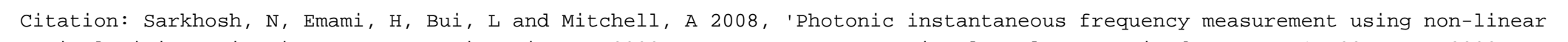

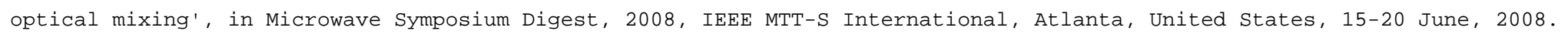

\title{
Photonic Instantaneous Frequency Measurement Using Non-Linear Optical Mixing
}

\author{
Niusha Sarkhosh, Hossein Emami, Lam Bui and Arnan Mitchell \\ Microelectronics and Material Technology Center, \\ School of Electrical and Computer Engineering, \\ Royal Melbourne Institute of Technology, \\ GPO Box. 2476V, Melbourne ,3001, Victoria ,Australia, \\ Email: niusha.sarkhosh@rmit.edu.au \\ Telephone: (613) 992-53250, Fax: (613) 992-52007
}

\begin{abstract}
In this paper we propose and demonstrate a photonically implemented instantaneous frequency measurement system. This system uses two differentially delayed modulated optical carriers that are mixed using a semiconductor optical amplifier. The output of the system includes a DC component that varies as a function of frequency. This can be used for frequency measurement using a low-cost DC photo-detector. Operation is demonstrated from $2-20 \mathrm{GHz}$.

Index Terms-Frequency measurement, optical frequency conversion, optical mixers, optical modulation, semiconductor optical amplifiers.
\end{abstract}

\section{INTRODUCTION}

Instantaneous frequency measurement (IFM) systems are important components of modern radar warning receivers. These systems identify the dominant frequency components of potential threat signals and thus provide an early indication of threat classification and also suggest frequency ranges in which to focus processing resources.

Traditional IFM devices can be implemented simply in the RF domain using interferometers formed from RF hybrids and RF delay lines[1]. These devices can be somewhat bulky and rely on multi-octave RF componentry which can be difficult to implement. Usually banks of these devices are required and they are positioned close to the receiving antenna to ensure the best sensitivity and dynamic range.

In recent years, microwave photonics has been investigated as a means of reducing the bulk of signal processing systems required at the receiving antenna. The general concept is to use broadband, low noise optical modulators to convert RF signals into the optical domain, transmit them via optical fibre and then return them to the microwave domain using broadband photo-detectors.

It would be advantageous to implement an IFM system using photonics. A recent investigation into photonic IFMs has suggested using a network of Bragg gratings to achieve the IFM response [2]. A drawback of this system is that it relies on the use of broadband photo-detectors to receive the frequency measurement. Implementing banks of these devices would therefore be prohibitively expensive.

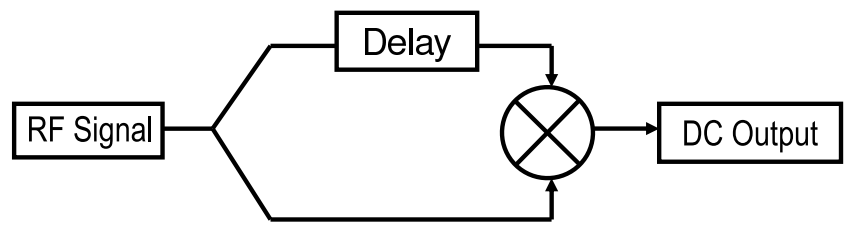

Fig. 1. Conceptual block diagram of the proposed IFM

In this paper we propose and demonstrate an alternate photonic IFM approach. We utilize a semiconductor optical amplifier as an all-optical mixer. Utilizing this component as part of our IFM system we have succeeded in achieving frequency measurement from $2-20 \mathrm{GHz}$ and require only DC photo-detection.

\section{THEORY}

In this section, a configuration producing a frequency dependent DC signal from an RF signal is proposed. The block diagram of the system is presented in Fig. 1. An RF signal was divided by two equal portions. The signals are delayed with respect to one and other and then multiplied together.

Mathematically, the output of Fig. 1 can be written

$$
V_{\text {out }}(t)=\frac{1}{2} V_{0}^{2} \cos \Omega t \cos \Omega(t-\tau)
$$

or

$$
V_{\text {out }}(t)=\frac{1}{4} V_{0}^{2}[\cos (2 \Omega t-\Omega \tau)+\cos \Omega \tau]
$$

where $V_{0}$ and $\Omega$ are the amplitude and angular frequency of the RF signal respectively and $\tau$ is the relative delay between the two paths. As it can be seen $V_{\text {out }}(\mathrm{t})$, has a frequency dependent DC portion of $\frac{1}{8} V_{0}^{2} \cos \Omega \tau$.

Having established a relation between the frequency and output DC component, we are now able to design an instantaneous frequency measurement system which is able to predict the frequency of an RF signal using only the DC component of the output. 


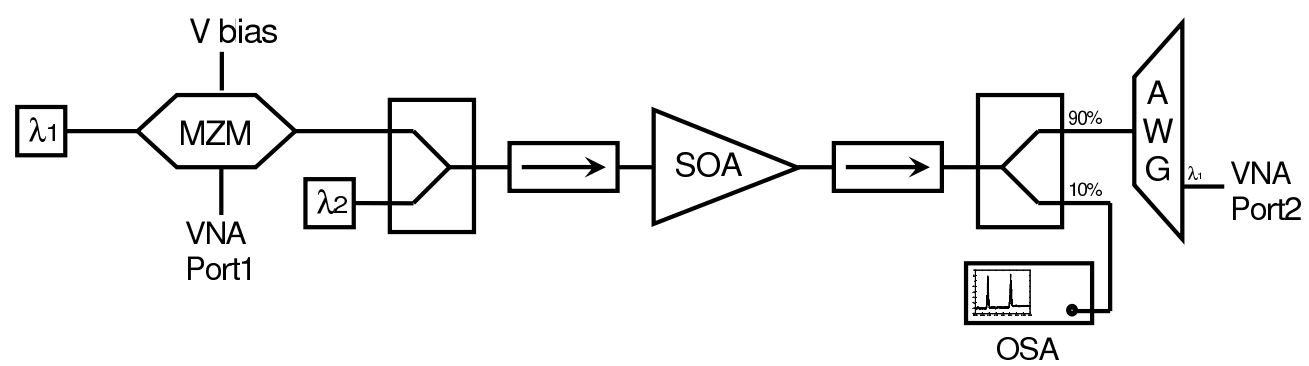

Fig. 2. Block diagram of the system used to characterize the SOA.

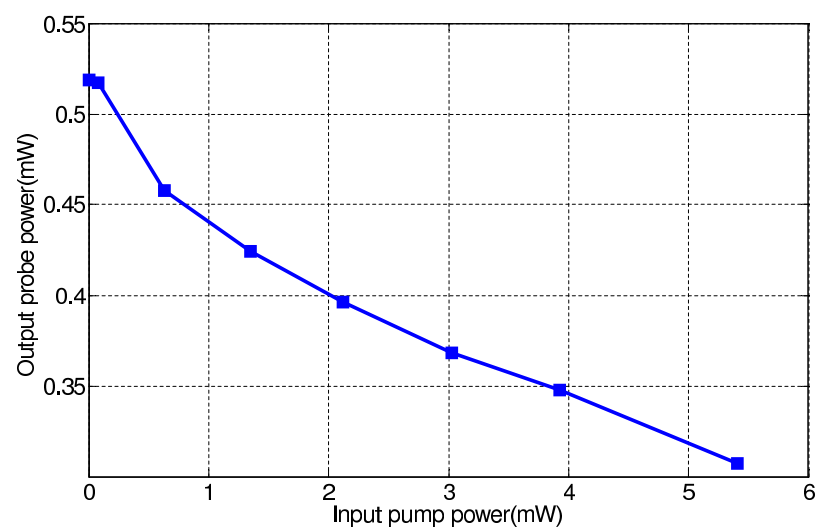

Fig. 3. DC response of the SOA.

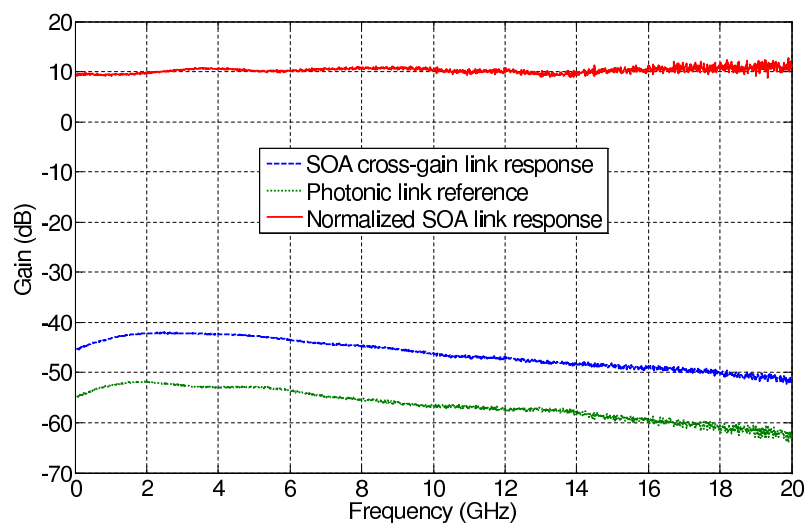

Fig. 4. Frequency response of the SOA: Photonic Link gain reference response; cross-gain frequency response; normalized cross-gain response.

\section{Multiplication in the Optical Domain Using A SEMiconductor Optical AMPLIFIER}

To achieve broad bandwidth, long transmission range and reduced bulk we wish to implement our IFM system using photonic components. Modulation, time delay and detection are all straightforward in the optical domain. All-optical multiplication can be achieved using nonlinear optical components. In this investigation multiplication is achieved using a semiconductor optical amplifier (SOA) [3]-[4]. Before proceeding with our IFM system implementation it will be necessary to characterize the response of an SOA when used as an all optical mixer.

Fig.2 presents the experimental setup used to characterize the nonlinear response of the SOA. Two laser sources provide two optical carriers at wavelengths $\lambda_{1}=$ $1551.85 \mathrm{~nm}$ and $\lambda_{2}=1553.81 \mathrm{~nm}$. Carrier $\lambda_{1}$ is transmitted through a high-speed Mach-Zehnder modulator (MZM) and then combined with carrier $\lambda_{2}$ using a simple $3 \mathrm{~dB}$ power combiner. The two optical carriers are then transmitted through the semiconductor optical amplifier (Kamelian, SOA-NL-H1-C-FA) where they are mixed by the SOA nonlinear response. Optical isolators are used on both sides of the SOA to prevent oscillation. A small portion $(10 \%)$ of output is tapped to an optical spectrum analyzer (OSA) to enable monitoring of the output optical wavelengths. The remaining optical power is separated into its wavelength components using an arrayed waveguide grating (AWG) and the signal on carrier $\lambda_{2}$ is monitored using a broadband photo-detector.

The first characterization experiment aimed to establish the DC mixing response of the SOA by measuring the output power on carrier $\lambda_{2}$ as a function of the input power on carrier $\lambda_{1}$. The input power at the SOA on carrier $\lambda_{2}$ was fixed at $5 \mathrm{~mW}$ and the modulator bias was used to adjust the input power of carrier $\lambda_{1}$. The output power on carrier $\lambda_{2}$ was measured using a power meter.

Fig. 3 presents the measured DC response. The gradient corresponds to the mixing gain. We choose to operate at a pump power of $5 \mathrm{~mW}$ where the gradient is most linear.

Next, we wished to establish the frequency response of the SOA mixing response. The configuration of Fig. 2 was again used. An RF signal from a vector network analyzer (VNA) was input to the MZM and the output was received by a broadband photodetector and analyzed using the VNA. The system was first calibrated by measuring the photonic link response of carrier $\lambda_{1}$ with the SOA component removed. The SOA was returned and the response of the signal transferred to carrier $\lambda_{2}$ was measured.

The RF mixing response of the SOA is presented in Fig. 4. The gain level is high and is quite flat over a broad frequency range from $2-20 \mathrm{GHz}$.

The SOA characteristics indicate that it is a good candidate for the mixing component for a photonic implementation the IFM of Fig. 1. 


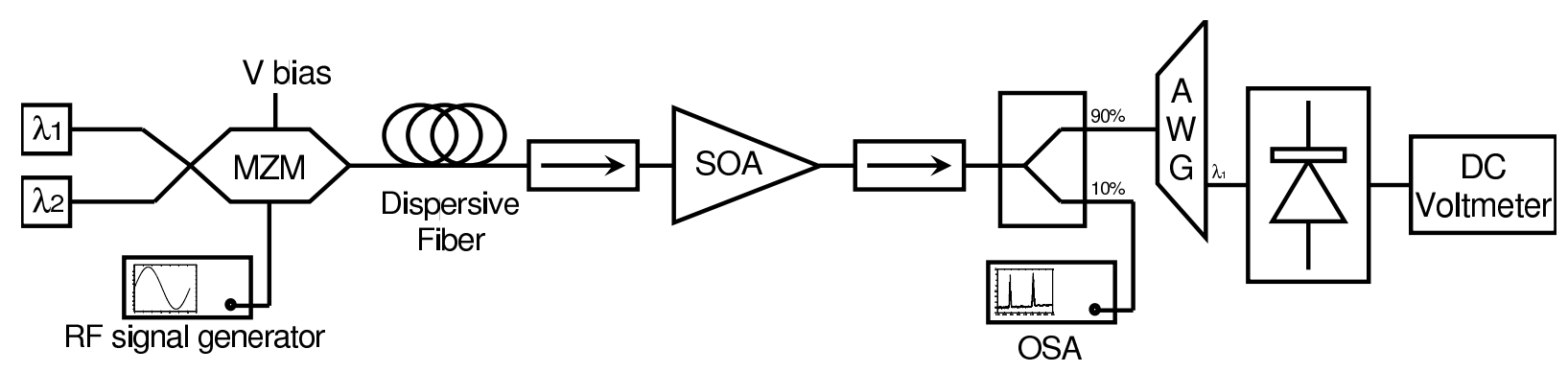

Fig. 5. Photonically implemented IFM configuration

\section{Photonically Implemented IFM}

The photonic IFM implementation is presented in Fig. 5. Compared with Fig. 2, a 2x1 MZM is used to combine and modulate both optical carriers; a length of dispersive fibre is used to achieve a relative time delay; and only the DC component is analyzed at the output.

Before measuring this system we predict its performance. Considering the frequency response of each component of the system, the DC output will be:

$$
V(f)=\sqrt{G(f)} \cos (2 \pi f \tau)]+V_{D C}
$$

where $f$ is the frequency of the signal and $G(f)=$ $G_{M Z M}^{2}(f) G_{S O A}(f) G_{P D}$ is the gain response of the system where $G_{M Z M}(f)$ is MZM RF gain response (a simple quadratic function), $G_{S O A}(f)$ is the conversion gain response of the SOA (Fig. 4), and $G_{P D}$ is the DC gain of the photo-detector. Since both carriers are modulated, thier product includes the modulator response squared.

Fig. 6 present the response predicted by Eq. (3). The sinusoidal response required for frequency measurement is evident. The gradually decreasing amplitude is mainly due to the quadratic MZM frequency response.

The system of Fig. 5 was implemented using a dispersive fibre (SMF28) of length $4.478 \mathrm{~km}$ which corresponded to a relative time delay of $\tau=149.165 \mathrm{ps}$ between the two optical carriers. Fig. 6 shows the DC component measured by the digital voltmeter as a function of frequency along with the predicted results. Excellent agreement between measurement and predicted results are evident.

The results of Fig. 6 can be inverted using Eq. (3) to achieve frequency measurement as shown in Fig. 7. Due to the oscillating nature of the response of Fig. 6, there are six distinct bands within which frequency measurement is unambiguous. The operation of this device is thus limited to within one of these bands. The bandwidth can be extended simply by reducing the length of the dispersive fibre. This improved bandwidth would come at the expense of reduced frequency sensitivity.

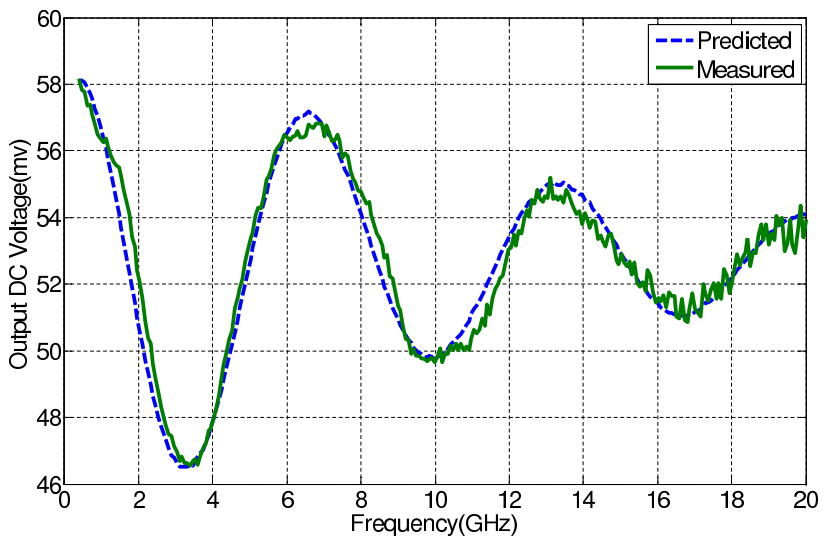

Fig. 6. Measured and predicted IFM response

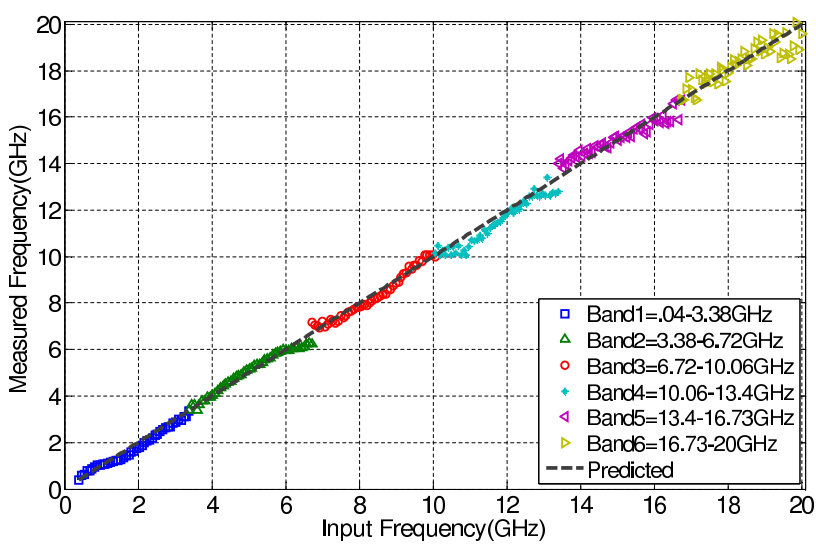

Fig. 7. IFM frequency response interpreted as frequency measurement

\section{REFERENCES}

[1] H. Gruchala, and M. Czyzewski, "The instantaneous frequency measurement receiver in the complex electromagnetic environment," 2004 MIKON Int. Conf., vol. 1, pp. 155-158, May 2004.

[2] L. V. T. Nguyen, and D. B. Hunter, "A photonic technique for microwave frequency measurement," IEEE Photon. Tech. Lett., vol. 18, no. 10, pp. 1188-1190, May 2006.

[3] J. Capmany, S. Sales, D. Pastor, A. Martinez, and B. Ortega "Wavelength conversion of SCM signals using semiconductor optical amplifier: theory, experiments, and applications," J. Lightwav. Tech., vol. 21, no. 4, pp. 961-972, April 2003.

[4] S. J. B. Yoo, "Wavelength conversion technologies for WDM network applications," J. Lightwav. Tech.., vol. 14, no. 6, pp. 955-966, June 1996. 